\title{
Salute renale per tutti e ovunque: riflessioni al femminile sul tema centrale della Giornata Mondiale del Rene 2020
}

\author{
Anna Laura Fantuzzi ${ }^{1}$, Elisa Berri' ${ }^{2}$, Lida Tartaglione ${ }^{3,4}$, Rossella Giannini ${ }^{5}$, Sara Dominjanni ${ }^{6}$, Silvia Porreca \\ ${ }^{1}$ Dietista già Coordinatore Nutrizione e Dietetica Aziendale, AUSL Modena, Comitato Scientifico ASAND (Associazione Scientifica \\ Alimentazione Nutrizione e Dietetica dei Dietisti Italiani), Reggio Emilia - Italia \\ ${ }^{2}$ Dietista, Nutrizione e Dietetica Aziendale, AUSL Modena, Modena - Italia \\ ${ }^{3}$ Dirigente Medico, UOC Nefrologia e Dialisi, ASL RM5, P.O. Tivoli, Roma - Italia \\ ${ }^{4}$ Dirigente Medico, Dipartimento Medicina Traslazionale e di Precisione, Università La Sapienza, Roma - Italia \\ ${ }^{5}$ Dietista, SSD Malattie del Metabolismo e Nutrizione Clinica, Azienda Ospedaliero-Universitaria Policlinico di Modena, Modena - Italia \\ ${ }^{6}$ Dirigente Medico, UOC Nefrologia e Dialisi Ospedale Sant'Eugenio, ASL Roma 2, Roma - Italia \\ ${ }^{7}$ Dirigente Medico, U.O. di Nefrologia e Dialisi, P.O. Della Murgia “F. Perinei”, Altamura (BA), e ASL Bari, Bari - Italia
}

\begin{abstract}
Kidney health for everyone and everywhere: reflections to the female on the central theme of the World Kidney Day 2020

The goal of the World Kidney Day 2020 (WKD) is "the promotion of public health by ensuring universal, equitable and sustainable access to care, making access to health for all people and social categories equitable". Chronic Kidney Disease (CKD) is a complex and "demanding" health problem for patients and professionals, particularly in the more advanced stages of the disease. In addition to factors such as the so-called systemic conditions and risk factors, sex-related factors intervene in women, which can further complicate the CKD trend. For these reasons, one of the objectives of the World Kidney Day is that prevention and early treatment are included in the Universal Health Coverage (UHC) and that common strategies are implemented to promote awareness of this pathology. The woman has always played a central role in the "coordination" of family life and "taking care", a role transversal to the different ethnic groups, as evidenced also by the data relating to living kidney donation. This article gives a reflection on some issues related to prevention and treatment of CKD from a female perspective, such as what strategies to identify to implement prevention in the family starting from nutrition, such as the advice for the preparation of a diet plan respectful of the traditions of the country of origin, the identification and treatment of risk factors in order to implement strategies aimed at prevention dedicated to the female world and which style distinguishes the female approach to care.
\end{abstract}

Keywords: Eating, Ethnic groups, Kidney failure, Patient care, Primary prevention, Women

\section{Introduzione}

La malattia renale cronica (MRC) è una patologia molto diffusa nel Mondo, con una prevalenza crescente nella popolazione generale. La Giornata Mondiale del Rene (World Kidney Day), celebrata il 12 marzo 2020, aveva come tema

Received: April 6, 2020

Accepted: April 8, 2020

Published online: May 18, 2020

Indirizzo per la corrispondenza:

Anna Laura Fantuzzi

Via Bismantova 4

42019 Scandiano, Reggio Emilia - Italia

annalaurafantuzzi@gmail.com la "Salute Renale per tutti e ovunque: dalla prevenzione alla diagnosi, equo accesso ad assistenza e cura"; per noi impegnate quotidianamente nel panorama della malattia renale cronica è stato uno stimolo per una riflessione condivisa che riportiamo in questo lavoro, il cui obiettivo sarà quello di approfondire e sviluppare alcuni aspetti della tematica in un'ottica femminile. Secondo uno studio della Società Italiana di Nefrologia (SIN), la prevalenza della MRC in Italia è pari al $7 \%$ della popolazione ed è in progressivo e costante aumento, raggiungendo valori più elevati in presenza di comorbidità come ipertensione arteriosa, obesità e dislipidemia (1). Lo STUDIO CARHES (Cardiovascular risk in Renal patients of the Health Examination Survey), pubblicato nel 2010, ha reso disponibili per la prima volta dati di prevalenza della MRC su scala nazionale, che è risultata del $7,5 \%$ negli uomini e del $6,5 \%$ nelle donne, con una prevalenza degli stadi 1 e 2 
( $\pm 60 \%)$, rispetto agli stadi 3-5 $(40 \%)(2,3)$. Considerato che le donne rappresentano il 51,3\% della popolazione residente in Italia (dati ISTAT 2019), con una vita media di 80,1 anni per i maschi e di 84,6 per le femmine e un aumento progressivo della speranza di vita alla nascita delle femmine rispetto ai maschi (4), sarebbe interessante approfondire alcuni aspetti: 1) incidenza della MRC in tutti i suoi stadi nel sesso femminile; 2) come favorire l'accesso alle cure, apportare dei miglioramenti e definirne una quantificazione; 3) implementare un'attività costante di prevenzione rivolta al mondo femminile, il cui obiettivo sia l'aumento della consapevolezza di questa malattia.

Soffermandoci sulla differenza di genere, non è possibile quantificare la prevenzione e l'accesso alle cure per carenza di dati disponibili, invece vi è una maggiore probabilità di insorgenza di MRC nelle donne in relazione ad alcune specifiche condizioni. In dialisi, esse sembrerebbero in numero inferiore rispetto agli uomini in molti paesi, sebbene il rischio cardiovascolare e di ospedalizzazione appaia, generalmente, più elevato e, tra le donne sottoposte a trattamenti emodialitici, vi sia un numero inferiore di fistole arterovenose $(\operatorname{AVF})(5,6)$. Riguardo al trapianto e, in particolare, al tema della donazione le donne sono meno frequentemente riceventi (sia da vivente che da cadavere) e più sensibili alla donazione da vivente (5).

\section{La prevenzione inizia dall'alimentazione in famiglia: quali strategie per costruire un menù equilibrato e sostenibile?}

La prevenzione delle malattie renali si basa essenzialmente sull'apprendimento di corretti stili di vita, tra cui la creazione di modelli alimentari "sani", e sul controllo dei fattori intermedi di insorgenza e di progressione (7). Una strategia grazie alla quale la donna diventa un riferimento per la famiglia è la pianificazione di un menù gustoso ed equilibrato, nel rispetto delle esigenze di ciascuno e della tradizione. I principi cardine da seguire dovrebbero rispecchiare quelli della dieta mediterranea, secondo le Linee Guida italiane per una sana alimentazione (Tab. I) redatte dall'Istituto

Tabella I - Linee guida per una sana alimentazione: aspetti applicativi. Adattato dalle Linee Guida per una sana alimentazione, CREA. REV. 2018

\begin{tabular}{|c|c|}
\hline $\begin{array}{l}\text { Quali grassi, } \\
\text { quanti grassi }\end{array}$ & $\begin{array}{l}\text { Come comportarsi } \\
\text { - Preferire i grassi da condimento di origine vegetale (extravergine d'oliva e olio di semi) } \\
\text { - Moderare il consumo di grassi da condimento di origine animale (burro, lardo, panna..., ecc.) } \\
\text { - Moderare la quantità di grassi e oli da condimento } \\
\text { - Utilizzare i grassi da condimento preferibilmente "a crudo", limitando il ricorso alla frittura } \\
\text { - Evitare di riutilizzare dei grassi già cotti }\end{array}$ \\
\hline $\begin{array}{l}\text { Più cereali, legumi, } \\
\text { ortaggi e frutta }\end{array}$ & $\begin{array}{l}\text { Come comportarsi } \\
\text { - Consumare tutti i giorni pane, pasta, riso e altri cereali (anche integrali) } \\
\text { - Consumare tutti i giorni più porzioni di ortaggi e frutta fresca } \\
\text { - Aumentare il consumo di legumi sia freschi sia secchi }\end{array}$ \\
\hline $\begin{array}{l}\text { Quali proteine } \\
\text { scegliere }\end{array}$ & $\begin{array}{l}\text { Come comportarsi } \\
\text { - Non consumare più di } 2 \text { uova a settimana } \\
\text { - Aumentare la frequenza di consumo del pesce } \\
\text { - Preferire le carni magre e limitare il consumo di carne grasse } \\
\text { - Preferire latte o yogurt parzialmente scremati } \\
\text { - Scegliere formaggi freschi e consumare porzioni di misura ridotta }\end{array}$ \\
\hline $\begin{array}{l}\text { Zuccheri, dolci } \\
\text { e bevande } \\
\text { zuccherate: } \\
\text { meno è meglio }\end{array}$ & $\begin{array}{l}\text { Come comportarsi } \\
\text { - Preferire i dolci a ridotto contenuto di grassi e a maggiore contenuto di carboidrati complessi (prodotti da forno } \\
\text { della tradizione italiana: biscotti, torte non farcite, ecc.) } \\
\text { - Utilizzare in quantità controllata i prodotti dolci da spalmare sul pane (marmellata, miele e creme) } \\
\text { - Limitare il consumo di dolciumi, specialmente quelli adesivi (caramelle, torroni, snack dolci) } \\
\text { - Tenere conto della quantità e della frequenza di consumo di alimenti e bevande dolci nella giornata } \\
\text { - Se si utilizzano bevande o alimenti dolcificati con edulcoranti, controllare di non superare il dosaggio consigliato }\end{array}$ \\
\hline $\begin{array}{l}\text { Il sale? Meno è } \\
\text { meglio }\end{array}$ & $\begin{array}{l}\text { Come comportarsi } \\
\text { - Nella preparazione dei cibi, non eccedere con il sale } \\
\text { - Dare la preferenza al pane toscano e non eccedere con il consumo degli alimenti ricchi di sale (scatolame, } \\
\text { insaccati, ecc.) } \\
\text { - Limitare l'uso del sale in tavola } \\
\text { - Utilizzare erbe aromatiche e spezie per insaporire i cibi } \\
\text { - Controllare le etichette }\end{array}$ \\
\hline
\end{tabular}


Tabella I - (Continua)

\begin{tabular}{|c|c|}
\hline $\begin{array}{l}\text { Liquidi, bere } \\
\text { un adeguato } \\
\text { quantitativo }\end{array}$ & $\begin{array}{l}\text { Come comportarsi } \\
\text { - Bere ogni giorno acqua in abbondanza } \\
\text { - Bere bevande alcoliche il meno possibile } \\
\text { - Evitare l'assunzione di alcol durante l'età evolutiva, in gravidanza e nell'allattamento e ridurla nell'anziano } \\
\text { - Evitare l'alcol, se si assumono farmaci }\end{array}$ \\
\hline & $\begin{array}{l}\text { Perché variare l'alimentazione? Per garantire l'apporto di tutti i principi nutritivi } \\
\text { Nessun alimento è vietato: l'importante è variare e controllare la quantità di consumo, } \\
\text { cioè le porzioni di consumo degli alimenti }\end{array}$ \\
\hline Come variare & $\begin{array}{l}\text { Scegliere porzioni di cibi dai diversi gruppi di alimenti, alternandoli nei vari pasti della giornata. } \\
\text { - Cereali e tuberi } \\
\text { Pane, pasta, riso, mais, avena, orzo, patate } \\
\text { Scegliere, in questo gruppo, 2-4 porzioni al giorno (1 porzione per ogni pasto) } \\
\text { - Frutta e ortaggi } \\
\text { Scegliere, in questo gruppo, } 5 \text { porzioni al giorno } \\
\text { Le verdure possono essere utilizzate anche per preparare gustosi primi o secondi piatti o piatti unici } \\
\text { - Latte e derivati } \\
\text { Compresi yogurt e formaggi } \\
\text { Scegliere, in questo gruppo, 1-2 porzioni al giorno } \\
\text { - Carne, pesce, uova } \\
\text { Compresi anche i legumi secchi } \\
\text { Scegliere, in questo gruppo, 1-2 porzioni al giorno } \\
\text { - Grassi da condimento } \\
\text { Scegliere, in questo gruppo, 1-3 porzioni al giorno }\end{array}$ \\
\hline $\begin{array}{l}\text { Sostenibilità } \\
\text { delle diete: tutti } \\
\text { possiamo } \\
\text { contribuire }\end{array}$ & $\begin{array}{l}\text { Come comportarsi } \\
\text { - Mettere in opera tutte le strategie possibili di lotta allo spreco alimentare perché molto si può fare anche a livello } \\
\text { domestico. Per produrre cibo che non verrà consumato vengono inutilmente utilizzate risorse naturali e generati } \\
\text { emissioni nell'atmosfera e rifiuti } \\
\text { - Programmare la spesa alimentare cercando di non fare scorte che non si riescono a smaltire, per evitare che } \\
\text { parte della spesa vada a male e debba essere eliminata } \\
\text { - Riporre con attenzione la spesa: gli alimenti più "nuovi", con una data di scadenza più lontana, devono essere } \\
\text { posti in posizione meno visibile nel frigorifero o nella dispensa, mentre davanti devono essere messi quelli più vec- } \\
\text { chi, per consumarli prima ed evitare che vadano sprecati } \\
\text { - Riciclare gli avanzi in nuove ricette, mangiare il giorno dopo quello che è avanzato, purché secondo le regole } \\
\text { indicate nella direttiva "la sicurezza degli alimenti dipende anche da te". Non sprecare e insegnare a non } \\
\text { sprecare vogliono dire fare cultura del valore del cibo }\end{array}$ \\
\hline
\end{tabular}

Nazionale di Ricerca per gli Alimenti e la Nutrizione (8). II primo messaggio è che "nessun alimento è vietato, l'importante è variare e controllare la quantità di consumo", cioè le porzioni degli alimenti (7). Di seguito, si riassumono alcuni principi fondamentali.

Privilegiare prodotti di origine vegetale: consumare 5 porzioni al giorno tra frutta e verdura. Le verdure possono essere utilizzate anche come ingredienti per primi e secondi piatti o per i piatti unici. L'evidenza disponibile riporta che il consumo di frutta e verdura sia un fattore di protezione nei confronti di sovrappeso e malattie cronico-degenerative, poiché apportano acqua, fibra, vitamine, minerali e sostanze bioattive. Inoltre, la presenza di frutta e verdura permette di ridurre la densità energetica della dieta perché il loro potere saziante è particolarmente elevato (8).

In ogni pasto non deve mai mancare una porzione di cereali, come pasta, riso, farina di mais, pane e prodotti da forno; a questo gruppo appartengono anche le patate. Per quanto riguarda i condimenti dei primi piatti, preferire sughi di verdure e/o legumi. Questi ultimi dovrebbero essere inseriti almeno 2 volte a settimana e utilizzati come ingredienti di piatti unici, come previsto da molte ricette della tradizione (pasta e ceci, riso e piselli), in quanto apportano amido, fibra, proteine vegetali, vitamine, minerali e altre sostanze di interesse per la salute. La famiglia dei cereali comprende anche orzo, sorgo, miglio, avena, segale e farro, recentemente riscoperti. Cereali e derivati sono la fonte principale di carboidrati, che dovrebbero essere, per la maggior parte, complessi (amidi) e, per una parte minore, semplici (zuccheri); essi sono anche una buona fonte di fibra insieme a frutta, verdura e legumi (8).

Fare attenzione alle proteine. I dati emergenti tra individui e popolazione suggeriscono che una dieta ricca di proteine può portare a un rischio maggiore di MRC de novo o ad accelerare la progressione di una MRC preesistente (9). Considerare la frequenza di consumo e le porzioni degli alimenti a 
elevato contenuto di proteine, in particolare di origine animale: carne, pesce, uova, salumi, latte e latticini. Ad esempio, non consumare un doppio secondo, evitare di aggiungere formaggio e salumi a fine pasto oppure ridurre le porzioni quando si consumano carne o pesce o salumi come antipasto in aggiunta al secondo piatto. Consumare piatti unici utilizzando questi alimenti come ingredienti nelle preparazioni (pasta al ragù di carne o al sugo di pesce). In linea generale, moderare il consumo di carne, sostituendola più spesso con cereali e legumi (8).

Controllare la quantità e la qualità dei grassi. Preferire I'olio extravergine di oliva, sia per la cottura che come condimento, utilizzando metodi e strumenti di cottura che ne limitino la quantità, e aggiungerlo preferibilmente a crudo. I grassi danno sapore, contribuendo all'aroma e alla piacevolezza dei cibi, perciò, nel mondo occidentale, vengono consumati in maniera eccessiva (8).

Fare attenzione al sale, ingrediente di cui si abusa, spesso inconsapevolmente. A tal proposito è fondamentale limitare I'uso della saliera a tavola, utilizzare alimenti freschi, implementare l'uso di aromi e spezie, limitare gli alimenti a elevato contenuto intrinseco di sale (salumi, formaggi) e preferire il pane senza sale (8).

Bere un adeguato quantitativo di liquidi, almeno 2 litri al giorno, quantitativo che può variare in relazione a esercizio fisico, clima, condizioni di salute, gravidanza e allattamento, sesso ed età. Assecondare il senso di sete tentando di anticiparlo, ricordando che anziani e bambini sono esposti maggiormente al rischio di disidratazione. Una buona idratazione è un fattore di prevenzione e terapia per la calcolosi urinaria, la stipsi, le cistiti e l'iperglicemia nei pazienti diabetici. La disidratazione, anche quando di lieve entità, può essere un fattore di rischio o di aggravamento di varie patologie (8).

Per passare dalla teoria alla pratica, trasformando in piatti accattivanti e gustosi le indicazioni per una sana alimentazione, ecco alcuni suggerimenti: a) costruire un menù settimanale che possa accontentare le preferenze di tutti; b) pianificare il menù per evitare la monotonia e la ripetitività della dieta quotidiana; c) confezionare piatti con ricette semplici e veloci; d) adattare le ricette della tradizione alle esigenze del o dei familiari che hanno problemi di salute; e) preferire l'acquisto e il consumo di alimenti prodotti localmente e certificati. Fare la spesa seguendo una lista degli acquisti preparata a casa aiuta a evitare gli sprechi e a risparmiare, guadagnando in salute per se stessi, per la famiglia e per l'intera società.

\section{La prevenzione inizia dall'identificazione e dalla cura dei fattori di rischio: quali sono quelli sesso-specifici di sviluppare una malattia renale cronica?}

L'influenza del sesso su insorgenza, progressione e fenotipo clinico di diverse patologie di pertinenza nefrologica non è ancora chiara e solo recentemente la ricerca (sia clinica che sperimentale) si sta dedicando a tale argomento. Infatti, è diventato sempre più chiaro come le differenze, sia biologiche (quelle dovute al sesso, ovvero al quadro ormonale differente) che di genere (gli aspetti socioculturali riguardanti il ruolo che uomini e donne svolgono a livello sociale e le relative relazioni), possano influenzare la prevalenza, la progressione e la prognosi della MRC; la stima della sua prevalenza è centrale nel disegnare adeguate strategie di prevenzione. Grazie a criteri precisi di definizione della MRC delle Linee Guida KDOQI del 2002 (10), i dati relativi alla prevalenza sono in costante aumento. È evidente che non esistono dati uniformi riguardo alla prevalenza della MRC nei diversi paesi (1114). Tale disomogeneità sembrerebbe legata a una differente modalità di stima del filtrato glomerulare e del dosaggio della creatinina e a un'eterogeneità del periodo di raccolta dati e delle popolazioni studiate. Tuttavia, nonostante la prevalenza della MRC sia risultata diversa in molti studi, quasi invariabilmente si osserva una maggiore prevalenza tra le donne che tra gli uomini. La spiegazione potrebbe essere quella che considera l'aspettativa di vita delle donne e la riduzione fisiologica del filtrato con l'età (15). Inoltre, spesso, le formule utilizzate per stimare il filtrato glomerulare renale, come la Chronic Kidney Disease Epidemiology Collaboration (CKDEPI) o la Modification of Diet in Renal Disease (MDRD), non sono sviluppate in popolazioni con un'età appropriata e di diversa etnia. In effetti, quando, in popolazioni specifiche, si misura il filtrato glomerulare con metodiche più precise, come la clearance dello iohexolo, la differenza tra i due sessi risulta attenuata, se si corregge la clearance per l'area di superficie corporea (16). Dal momento che gli uomini tendono ad avere una maggiore superficie corporea e la massa renale è direttamente proporzionale a questa, correggere il filtrato per una superficie corporea fissa non sempre risulta la metodica più adeguata.

A conferma di ciò, gli studi che analizzano l'insorgenza e l'andamento della MRC suggeriscono come il sesso maschile rappresenti un fattore di rischio per una più rapida progressione della stessa. In particolare, uno studio di registro giapponese che ha arruolato ben 107.192 pazienti (51.122 uomini e 56.070 donne), seguiti per circa dieci anni, ha mostrato come l'incidenza cumulativa dell'insufficienza renale cronica terminale (End Stage Renal Disease, ESRD) risultava sempre più bassa nelle donne (sia in epoca preche in epoca post-menopausale) e che l'aumento del rischio con il progredire dell'età sembrava spostato di qualche anno nelle donne rispetto agli uomini. II sesso maschile risultava, quindi, un fattore di rischio di ESRD (adjusted odds ratio $1,41,95 \%$ intervallo di confidenza 1,04-1,92) (17). Tale dato si è confermato anche in altri studi di registro. Nello studio tedesco PREVEND, la riduzione del filtrato glomerulare annuo è risultata essere $-0,55 \pm 1,47$ negli uomini vs $-0,33$ $\pm 1,41 \mathrm{~mL} / \mathrm{min} / 1,43 \mathrm{~m}^{2}$ nelle donne (18). La discrepanza poteva essere spiegata in parte con lo stato ormonale e, in 
particolare, poteva essere attribuita al ruolo antiapoptotico e proliferativo che hanno gli estrogeni sulle cellule tubulari renali (19), in parte con differenze nello stile di vita (incluse le abitudini alimentari, con particolare riguardo all'intake proteico e di sale, all'abitudine tabagica e all'assunzione di alcol). Diversi studi hanno indicato che il rischio di ESRD con necessità di inizio di terapia sostitutiva renale è più alta negli uomini che nelle donne. Per un paziente di 40 anni di sesso maschile negli USA, in Canada e in Europa, tale rischio si aggira intorno al 3,3\%, al 2,7\% e all'1,4\%. In rapporto, nelle donne il rischio è inferiore e, rispettivamente, del $2,2 \%$, dell' $1,8 \%$ e dello $0,7 \%$ (20-22). La spiegazione potrebbe essere la maggiore progressione della MRC segnalata negli uomini, pur non escludendo l'ipotesi che le donne non abbiano le stesse opportunità di iniziare il trattamento dialitico, che hanno una maggiore propensione a optare per la terapia conservativa, fino all'ipotesi di una maggiore mortalità pre-ESRD. Nella popolazione italiana, i dati più recenti del già citato studio CARHES mostrano come non ci siano differenze in termini di prevalenza di MRC agli stadi $1-5$, che si aggirava intorno al $7 \%$ sia nelle donne che negli uomini. Tuttavia, l'analisi per stadi di filtrato glomerulare e gradi di proteinuria ha evidenziato come, nel sesso maschile, c'è una più alta prevalenza di MRC agli stadi 1-2 con fenotipo albuminurico, che non si mantiene negli stadi più avanzati e che potrebbe essere spiegata da una maggiore presenza di ipertensione arteriosa, diabete, fumo e sovrappeso (22).
Per tutti i pazienti, l'analisi multivariata selezionava, quali fattori di rischio indipendenti di MRC, età, diabete, fumo, ipertensione arteriosa e malattia cardiovascolare (23). Per le donne, i fattori specifici sesso-correlati di rischio relativo sono dovuti a una maggiore probabilità di infezioni delle vie urinarie, dovuta alla diversa anatomia, mentre la gravidanza rappresenta un fattore di rischio specifico. Nei paesi più poveri, nel $15-25 \%$ delle donne si osserva un'insufficienza renale acuta, da cui deriva un rischio aumentato di mortalità materna e fetale (24). La pre-eclampsia e l'ipertensione gestazionale aumentano il rischio di ipertensione e di MRC nelle donne che le hanno sviluppate durante la gravidanza (25). L'obesità, l'ipertensione arteriosa, la sindrome metabolica e il diabete rappresentano fattori di rischio classici sia di MRC che di pre-eclampsia, innescando, così, un circolo vizioso di difficile risoluzione. L'obesità è riscontrata più frequentemente nelle donne, rendendole, così, a rischio di nefropatie specifiche (glomerulopatie obesità-correlate) e di progressione di MRC verso l'ESRD (26). Per quanto riguarda le malattie glomerulari, è nota una maggiore prevalenza tra il sesso femminile di nefrite lupica, nefropatia da membrana sottile e nefropatia da IgA (27). I meccanismi patogenetici alla base di queste differenze epidemiologiche ancora non sono chiari e, probabilmente, sono da correlare con gli effetti immunomodulatori degli ormoni sessuali endogeni. La Figura 1 rappresenta i principali fattori coinvolti nella patogenesi della MRC legati al sesso.

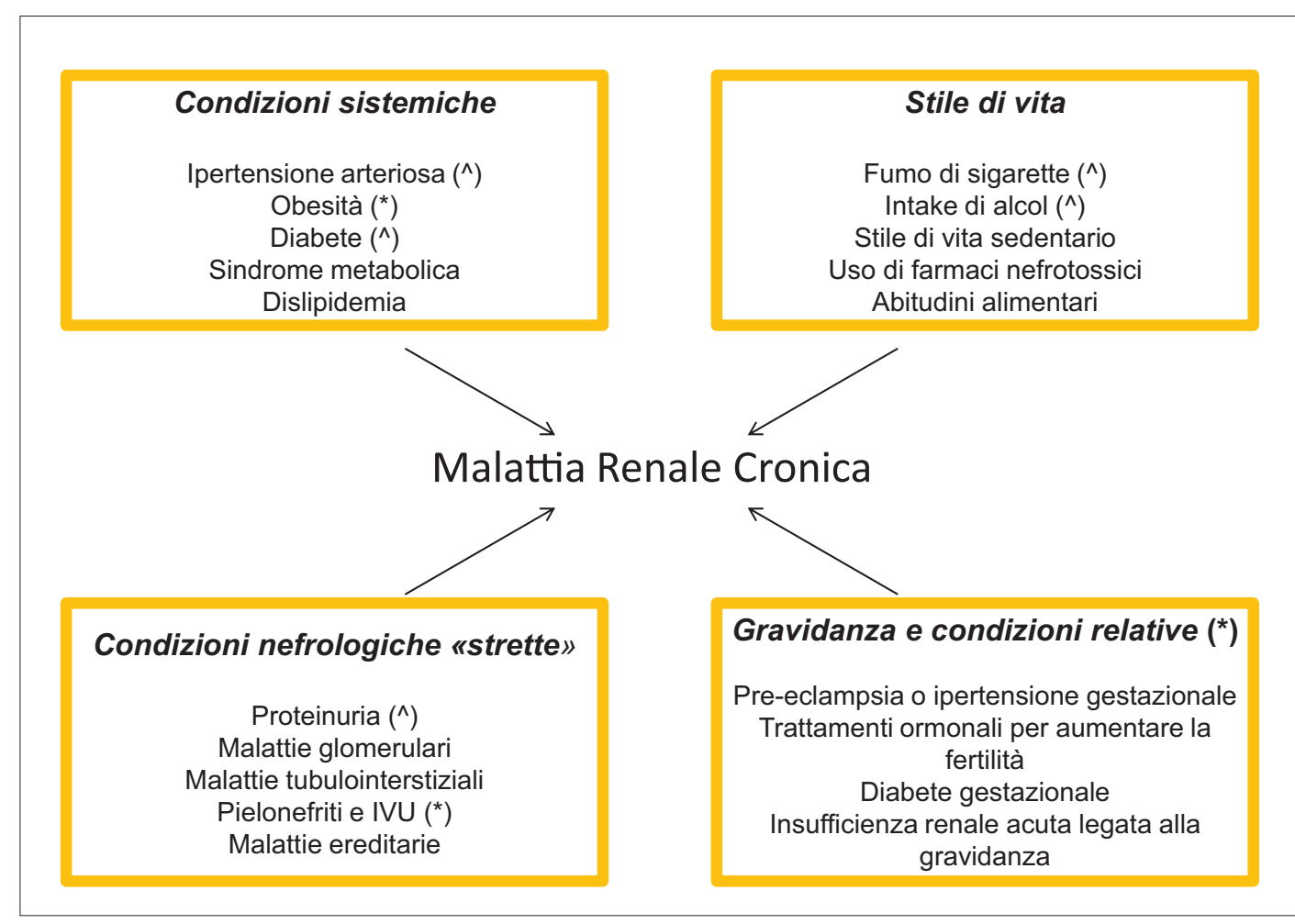

Fig. 1 - Principali fattori $d i$ rischio di sviluppo di MRC. $\mathrm{IVU}=$ infezione delle vie urinarie. $\left({ }^{\wedge}\right)$ più frequenti nel sesso maschile, $\left({ }^{*}\right)$ più frequenti nel sesso femminile. 


\section{Il confronto di genere nell'approccio al paziente con MRC: dal "to cure" al "to care"}

La percezione dello stato di salute sta acquisendo sempre più importanza sia nella valutazione dell'impatto che una determinata patologia ha sulla qualità di vita dei pazienti sia nel definire gli obiettivi terapeutici che per giudicare l'efficacia del trattamento. Le malattie croniche possono compromettere a lungo la qualità della vita delle persone, oltre a rappresentare un'importante causa di morte. Diventa, dunque, di rilevante importanza la qualità dell'approccio terapeutico. Il concetto di "to cure" spesso si accompagna a una rappresentazione iconografica del medico, uomo, possibilmente di una certa età o con segni che ne denotino l'esperienza clinica e che viene, nell'immaginario comune, identificato come I'individuo idealmente più indicato a somministrare tale cura. Ormai da tempo al concetto di "to cure" si è affiancato quello di ben più valore storico e sociale del "to care", concetto che si declina tutto al femminile. C'è una predisposizione speciale in alcune persone nel "prendersi cura" di ciò che è caro, di ciò che ha valore per loro. Studi storici e archeologici hanno dato a questa inclinazione una connotazione femminile che risale alla notte dei tempi (ancor prima dell'Età del Bronzo). Da sempre, quindi, la sfera della cura, sia che si trattasse delle persone che degli animali o dell'abitazione, era di competenza delle donne. In qualità di depositarie del sapere attinente ai meccanismi della vita e della morte, esercitavano le loro conoscenze sul funzionamento della natura. Mentre la donna si concentrava sul mondo interno alla famiglia, alla tribù, l'uomo era proiettato verso l'esterno con la caccia e la difesa. Questo equilibrio è stato mantenuto per millenni, fino all'arrivo della cosiddetta civiltà della spada, che ha portato alle culture classiche e alla società come la conosciamo oggi.

Negli ultimi 40 anni, le donne, attraverso movimenti di genere, hanno affermato il diritto alla presa di parola e all'autodeterminazione, con la proposizione, tra l'altro, del concetto di salute dal punto di vista "di genere", dandone una definizione dinamica, inserita nel contesto sociale come bene comune. Le ricerche epidemiologiche confermano oggi quanto paghi l'investimento sulla promozione delle competenze delle donne, oltre a quanto siano fertili le loro potenzialità. In questa ottica, un ambulatorio di tipo multidisciplinare declinato al femminile, dedicato a una patologia cronica, come può essere quella renale, il cui peso sociale è ben noto, permette di certo una "visione" di salute pubblica più ampia, basata sul concetto di cura dinamica a $360^{\circ}$, mediante processi decisionali autonomi e consapevoli, secondo un approccio non direttivo ma orizzontale. La competenza del "to care", affiancata a quella del "to cure", declinata in un contesto multidisciplinare, dove l'approccio femminile della cura medica della salute come bene primario si affianca alla cura tutta femminile anche degli aspetti sociali e nutrizionali, permette di avere un approccio "di genere" costruttivo, basato sulle competenze di cui la figura della donna è da secoli depositaria.

\section{L'alimentazione nella MRC in fase conservativa: i consigli alle donne immigrate nel rispetto della cultura alimentare del paese di origine}

La GMR richiede che la prevenzione e il trattamento precoce delle patologie renali vengano inclusi nella Copertura Sanitaria Universale (CSU). L'obiettivo principale è quello di "promuovere la salute pubblica assicurando un accesso universale, equo e sostenibile alla cura, rendendo equo l'accesso alla salute a tutte le categorie sociali". L'annuario statistico ISTAT del 2019 riporta che la popolazione straniera residente in Italia era di 5.255 .503 unità, pari all' $8,7 \%$ del totale dei residenti, con un incremento del 2,2\% (circa 111.000 unità) rispetto all'anno precedente. La maggior parte degli stranieri proviene da Unione Europea (30,1\%), Europa centro-orientale extra UE (19,9\%), Africa settentrionale $(12,7 \%)$ e altri paesi asiatici (11,4\%). Oltre il 65\% dei nuovi permessi nel 2018 era stato rilasciato a cittadini non comunitari con meno di trenta anni. La struttura di genere rimane complessivamente bilanciata (51,7\% maschi vs $48,3 \%$ femmine), anche se con rilevanti squilibri all'interno delle diverse collettività (28). I dati relativi all'immigrazione evidenziano come il nostro paese sia un territorio multiculturale dove gli stranieri residenti accedono anche all'assistenza sanitaria. Ne consegue la necessità, per i professionisti sanitari, di avere una competenza specifica quale requisito per migliorare l'efficacia del proprio intervento. Nel paziente con MRC in terapia conservativa, la dieta ipoproteica assume una particolare importanza, e il dietista, nel porre al centro del proprio intervento il paziente e le sue esigenze, deve tenere conto della complessità della nutrizione, che assume anche un significato simbolico, sociale ed emotivo $(29,30)$. Il cibo è un tema centrale nella vita delle persone e la donna ha da sempre il ruolo fondamentale di health driver nel costruire e mantenere buone abitudini alimentari, ruolo trasversale a tutte le culture che diventa particolarmente importante quando la donna stessa o un suo familiare hanno un problema di salute dovuto alla riduzione della funzione renale. La gestione della terapia nutrizionale in relazione alla dieta ipoproteica diventa, quindi, un nodo cruciale dell'assistenza e richiede particolare attenzione. La presa in carico di un paziente straniero con malattia renale comporta la considerazione di criticità legate alla barriera linguistica che limita la comprensione delle informazioni ed è, quindi, fondamentale la presenza di un mediatore che possa trasferire le informazioni nel modo corretto, sia questo un familiare o una figura ausiliaria. Durante il colloquio, la valutazione delle abitudini alimentari è un requisito essenziale per la personalizzazione del piano dietetico (31) e anche per investigare aspetti caratterizzanti del comportamento alimentare della famiglia, come regole alimentari religiose, tipologia di alimenti consumati nelle occasioni speciali (p. es., festività), grado di integrazione e acculturazione alimentare (p. es., consumo di cibi italiani), credenze su alimentazione e salute e approvvigionamento di cibo (30). La cucina etnica del 
Tabella II - Piatti tipici con ingredienti di diversi paesi del mondo

\begin{tabular}{|c|c|c|c|}
\hline Nome del piatto & Caratteristiche & Ingredienti & Origine \\
\hline Ciorba de burta & $\begin{array}{l}\text { Primo piatto consumato nei giorni } \\
\text { di festa o al ristorante nei pranzi in } \\
\text { famiglia }\end{array}$ & $\begin{array}{l}\text { Trippa, ossa di vitello, carota, cipolla, prezzemolo, sedano, } \\
\text { tuorlo d'uovo, panna acida, aglio, farina, aceto, pepe nero, } \\
\text { peperoncino fresco, olio di semi di girasole }\end{array}$ & Romania \\
\hline \multirow[t]{2}{*}{ Burek } & $\begin{array}{l}\text { Piatto tipico che si consuma } \\
\text { accompagnato con lo yogurt }\end{array}$ & $\begin{array}{l}\text { Pasta sfoglia: farina, sale, olio, acqua; ripieno: carne } \\
\text { macinata, cipolla, pepe nero, sale, erbe; per condire: olio }\end{array}$ & Macedonia \\
\hline & $\begin{array}{l}\text { Esistono anche versioni con farcitura di } \\
\text { ricotta salata o patate }\end{array}$ & & \\
\hline Cous cous & $\begin{array}{l}\text { Si consuma per tutti i commensali in un } \\
\text { unico piatto utilizzando il cucchiaio o le } \\
\text { mani }\end{array}$ & $\begin{array}{l}\text { Cous cous cotto al vapore, carne di manzo o di agnello, } \\
\text { peperoni, peperoncino, cipolla, pomodoro, zenzero, curcuma, } \\
\text { carota, zucchine, patata, zucca, ceci, uvetta, olio, sale, burro }\end{array}$ & Marocco \\
\hline
\end{tabular}

continente africano prevede preparazioni come cous cous, fufu, jollof rice o tsebhi, con injera e hilbet (Tab. II), serviti in un unico piatto da portata e consumati con le mani: può essere utile educare le donne alla identificazione della porzione destinata al familiare con MRC. Nei paesi come Romania o Ucraina, dove vengono utilizzati panna o latte nei piatti tradizionali, come nel Boršči, può essere utile consigliare I'utilizzo di prodotti alternativi, come panna o latte vegetale (p. es., riso, soia o mandorla), al fine di ridurre l'assunzione di fosforo e proteine. La stessa modifica può essere consigliata ai pazienti provenienti da Filippine o Sri Lanka, dove vengono utilizzati il latte di cocco o lo yogurt nelle preparazioni. Un'adeguata formazione del dietista sulle abitudini alimentari multiculturali (cultural competence) è indispensabile per suggerire alle donne straniere strategie nutrizionali, tra cui le modifiche da apportare alle varie ricette e la possibilità di inserire alimenti/ingredienti specifici. La condivisione dei principali piatti tipici del paese di origine e la valutazione delle porzioni con strumenti come l'atlante fotografico degli alimenti consentono di fornire messaggi utili a orientare le scelte alimentari e il controllo delle porzioni, adeguando il trattamento dietetico alle esigenze "uniche" del paziente che si ha in cura. Se questo paziente è una donna, spesso si deve occupare anche dell'alimentazione della famiglia; per questo il lavoro del dietista diventa prezioso, poiché supporta la predisposizione di un'alimentazione adeguata per tutti.

\section{Conclusioni}

La MRC è una condizione "impegnativa", soprattutto nei suoi stadi finali e nella donna, oltre ai fattori come le condizioni cosiddette sistemiche (ipertensione, obesità, diabete, sindrome metabolica) e i fattori di rischio (fumo, alcol, alimentazione, sedentarietà), intervengono fattori sesso-correlati (anatomia dell'apparato urinario, pre-eclampsia, terapie ormonali) (24-27).

Argomenti centrali rimangono, da un lato, il ruolo della donna nel "coordinamento" della vita familiare e, dall'altro, il ruolo del "prendersi cura" ("to care") che essa ha avuto nelle generazioni e che mantiene anche nelle diverse etnie $(29,30)$. Basti pensare alla donazione di rene da vivente: la mamma dona al figlio e la moglie dona al marito, con numeri di donazione tra donne e uomini a favore delle prime $(54 \%$ vs 39\%) (5).

Per concludere, dal momento della prevenzione al momento della malattia conclamata, la donna svolge un ruolo chiave; parlare alle donne da donne, mettendosi dallo stesso lato della scrivania, siano esse affette da malattia renale o caregiver, può assicurare un efficace approccio bio-psico-sociale alla MRC.

\section{Disclosures}

Authors contribution: All authors contributed equally to this manuscript.

Conflict of interest: The authors declare no conflict of interest.

Financial support: This research received no specific grant from any funding agency in the public, commercial, or not-for-profit sectors.

\section{Bibliografia}

1. Provenzano M, Mancuso C, Garofalo C, De Nicola L, Andreucci M. Temporal variation of Chronic Kidney Disease's epidemiology. G Ital Nefrol. 2019;36(2).pii:2019-vol2.

2. De Nicola L, Dal Canton A. Epidemiology of chronic kidney disease in Italy: the CARHES study. G Ital Cardiol. 2010;11 (5 Suppl. 3):106S-108S. 
3. Documento di indirizzo per la Malattia Renale Cronica, Ministero della Salute, 2014 Novembre 5.

4. ISTAT. La Salute nelle Regioni Italiane - Bilancio di un decennio (2005-2015), 2019.

5. Piccoli GB, et al. Donne e Malattie renali: Riflessioni su WKD 2018. Giornale di Tecniche Nefrologiche e Dialitiche. 2018;30(1):7-11.

6. Artan AS, Kircelli F, Ok E, et al. Dialyzing women and men: does it matter? An observational study. Clin Kidney J. 2016;9(3): 486-93.

7. Percorso Diagnostico Terapeutico Assistenziale per le persone con malattia renale cronica. Dir. Gen. Cura della Persona, Salute e Welfare. Determinazione n. 9509/2019, int. succ., Regione Emilia Romagna.

8. Centro di Ricerca Alimenti e Nutrizione del Consiglio per la ricerca in agricoltura e l'analisi dell'economia agraria. Linee Guida per una Sana Alimentazione Italiana. Revisione 2018. Eds. CREA, Roma 2019.

9. Kalantar-Zadeh K, Kramer HM, Fouque D. High-protein diet is bad for kidney health - Unleashing the taboo. Nephrol Dial Transplant. 2020;35(1):1-4.

10. National Kidney Foundation. K/DOQI clinical practice guidelines for chronic kidney disease: evaluation, classification, and stratification. Am J Kidney Dis. 2002;39:S1-266.

11. Zhang L, Wang F, Wang $L$, et al. Prevalence of chronic kidney disease in China: a cross-sectional survey. Lancet. 2012;379 (9818):815-22.

12. Süleymanlar G, Utaş C, Arinsoy T, et al. A population-based survey of Chronic Renal Disease In Turkey-the CREDIT study. Nephrol Dial Transplant. 2011;26(6):1862-71.

13. Cirillo $M$, Laurenzi $M$, Mancini $M$, Zanchetti $A$, Lombardi C, De Santo NG. Low glomerular filtration in the population: prevalence, associated disorders, and awareness. Kidney Int. 2006;70(4):800-6.

14. Murphy D, McCulloch CE, Lin F, et al. Trends in prevalence of chronic kidney disease in the United States. Ann Intern Med. 2016;165(7):473-81.

15. Glassock R, Delanaye $P$, El Nahas M. An age-calibrated classification of chronic kidney disease. JAMA. 2015;314(6): 559-60.

16. Inker LA, Shafi T, Okparavero A, et al. Effects of race and sex on measured GFR: the multi-ethnic study of atherosclerosis. Am J Kidney Dis. 2016;68(5):743-51.
17. Iseki K, Iseki C, Ikemiya Y, Fukiyama K. Risk of developing endstage renal disease in a cohort of mass screening. Kidney Int. 1996;49(3):800-5.

18. Halbesma N, Brantsma AH, Bakker SJ, et al. Gender differences in predictors of the decline of renal function in the general population. Kidney Int. 2008;74(4):505-12.

19. Seppi T, Prajczer S, Dörler MM, et al. Sex differences in renal proximal tubular cell zhomeostasis. J Am Soc Nephrol. 2016; 27(10):3051-62.

20. Grams ME, Chow EK, Segev DL, Coresh J. Lifetime incidence of CKD stages 3-5 in the United States. Am J Kidney Dis. 2013; 62(2):245-52.

21. Turin TC, Tonelli M, Manns BJ, et al. Lifetime risk of ESRD. J Am Soc Nephrol. 2012;23(9):1569-78.

22. van den Brand JAJG, Pippias M, Stel VS, et al. Lifetime risk of renal replacement therapy in Europe: a population-based study using data from the ERA-EDTA Registry. Nephrol Dial Transplant. 2017;32(2):348-55.

23. De Nicola L, Donfrancesco C, Minutolo R, et al. Prevalence and cardiovascular risk profile of chronic kidney disease in Italy: results of the 2008-12 National Health Examination Survey. Nephrol Dial Transplant. 2015;30(5):806-14.

24. Gyamlani G, Geraci SA. Kidney disease in pregnancy: (Women's Health Series). South Med J. 2013;106(9):519-25.

25. Levin A, Tonelli M, Bonventre J, et al. Global kidney health 2017 and beyond: a roadmap for closing gaps in care, research, and policy. Lancet. 2017;390(10105):1888-917

26. Afshin A, Forouzanfar MH, Reitsma MB, et al. Health effects of overweight and obesity in 195 countries over 25 years. N Engl J Med. 2017;377(1):13-27.

27. O'Shaughnessy MM, Hogan SL, Thompson BD, et al. Glomerular disease frequencies by race, sex and region: results from the international kidney biopsy survey. Nephrol Dial Transplant. 2018;33(4):661-9. [Epub ahead of print]

28. ISTAT. Annuario Statistico Italiano 2019.

29. American Dietetic Association. Medical Nutrition Therapy: Chronic Kidney disease (non-dialysis). Chicago (CD-ROM). 2002.

30. Morana PP, Giannini R, Carboni E, Ghidoni M, Fantuzzi AL. La relazione terapeutica con il paziente migrante e la "cultural competence" del dietista: un contributo esperienziale. G It Diabetol Metab. 2017;37:17-22.

31. Fantuzzi AL, Bedogni G. Dieta ipoproteica e insufficienza renale cronica. Milano, UTET. 2001. 